\title{
Filterless and Semi-Filterless Solutions in a Metro-HAUL Network Architecture
}

\author{
Omran Ayoub, Shady Shehata, Francesco Musumeci, Massimo Tornatore \\ Department of Electronics, Information and Bioengineering, Politecnico di Milano, Italy \\ E-mails: \{firstname.lastname\}@polimi.it,
}

\begin{abstract}
Filterless optical networks based on broadcast-and-select nodes have been proven to be a cost-effective alternative to active photonic network solutions in core networks. However, due to the emergence of novel metro-based highbandwidth cloud-based services (e.g., Virtual Reality, 4K Video-on-Demand, etc.), filterless solutions have started to attract research attention also in the metro area. In this paper, we evaluate the performance of fully-filterless and semi-filterless (i.e., hybrid solutions between fully-filterless and active photonic architectures) optical-network architectures in terms of cost of network elements and spectrum utilization, in a metro-network scenario. Our evaluations show that, due to the ring-based hierarchical nature of metro networks, fully-filterless architectures tend to require excessive spectrum utilization as the broadcast effect spreads among all hierarchical rings. On the contrary, semi-filterless network architectures seem more promising due to the presence of filters that fend off the propagation of unfiltered channels. The results also show that it is more advantageous to deploy filters at nodes of the lower network levels than at nodes of higher network levels.
\end{abstract}

\section{INTRODUCTION}

Network traffic keeps steadily increasing fuelled by the growing adoption of bandwidth-hungry cloud services. Although this traffic increase is favoured by service providers, it does not generally introduce any extra profit for network operators, that are challenged to upgrade their network capacity while minimizing the network's capital expenditure (CapEx) and operational expenditure (OpEx). In this context, filterless optical network architectures [1], based on broadcast-and-select nodes, are emerging as a promising cost-effective solution.

Filterless optical networks attempt to reduce network cost by eliminating, or significantly reducing, the amount of active switching elements (e.g., Wavelength Selective Switch (WSS)-based Reconfigurable Optical Add-Drop multiplexers (ROADMs)), and replacing them with passive splitters and combiners. After having removed the active optical switches, reconfigurability of network connectivity in filterless networks will only be achieved exploiting the adaptability of modern coherent optical transmission. However, the elimination of active switching and filtering components enforces signal broadcast on all the outputs of the passive splitters, resulting in the transmission of optical signals over unintended links and in higher spectrum occupation with respect to an active photonic network. To mitigate the high spectrum requirements of fully-filterless solutions, while maintaining a significant reduction of network cost, the concept of semi-filterless networks, where some network nodes are filterless and others are filter-equipped, has also been proposed [2].

Filterless networks have already been investigated in core and regional networks [3]-[7]. Refs. [3][4] quantify the cost reduction and spectrum-occupation increase in filterless solutions and show that they can yield significant advantages in terms of power consumption. The Routing and Spectrum Assignment (RSA) problem in elastic filterless networks has been then formally introduced in Refs [5]-[6], showing again significant bandwidth savings compared to fixed-grid filterless solutions. More recently, Ref. [7] has investigated the RSA problem in filterless networks utilizing optical white-boxes, which are programmable switches that provide custom configuration of the interconnections between input/output ports and passive splitters and combiners. Significant spectrum savings can be achieved as the optical white boxes allow the reconfiguration of the node's fiber interconnections to accommodate changing traffic demands efficiently. All the studies mentioned so far motivate the deployment of filterless architectures as cost-effective solution for core networks. However, the increase in metro traffic (e.g., deriving from the ongoing deployment of nodes equipped with storage and computing capabilities, hosting Virtual Network Functions (VNFs) at metro-edge [8]) urges network operators to consider filterless optical solutions also in metro networks. Note that, due to the different characteristics of metro with respect to core, such as the network topology and traffic patterns, the conclusions drawn on core networks do not necessarily hold for metro networks. A recent study, Ref. [9], is the only to investigate filterless architectures in metro networks. The study presents a comparative cost analysis between a filterless and an active photonic network deployment over different traffic periods and shows that the filterless network deployment does not yield a significant cost reduction. However, some possible solutions to adapt metro network topology to filterless solution are sketched and left as future work.

In this work, we investigate the deployment of semi-filterless solutions (also referred to as hybrid solutions) in metro networks to mitigate the spectrum waste problem. We perform a comparative analysis of the deployment cost and spectrum usage of filterless, active photonic, and semi-filterless (hybrid) solutions in a metro network characterized by computing edge nodes capable of hosting and delivering services, following the vision of the H2020 Metro-Haul project [10]. 


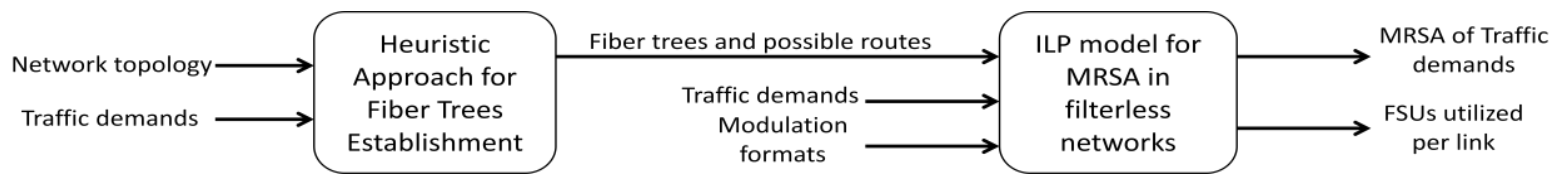

Figure 1 The overall framework of the filterless network design and resource allocation.

\section{FILTERLESS OPTICAL NETWORKS DESIGN}

The design approach used to perform comparison of filterless vs. semi-filterless solutions is structured in two steps. As a first step, we use a heuristic algorithm for the design of filterless optical networks based on dividing the network into edge-disjoint fiber trees, where each fiber tree denotes a set of interconnected optical fiber links and defines the interconnection between the passive splitters and combiners. The used approach is similar to that used in [4] but with a modification as we also consider the presence of filters in some nodes in the network. As a second step, we perform Modulation format, Routing and Spectrum Assignment (MRSA) of traffic demands with the objective of minimizing the overall network spectrum (overall framework is shown in Figure 1).

First step. Given the network topology (with the location of filters and filterless nodes in the network) and the traffic demands, our proposed heuristic algorithm establishes edge-disjoint fiber trees taking into consideration the "laser loop" and the "network connectivity" constraints. The laser loop constraint guarantees that no closed loops are present in the interconnection of the fibers, while the network connectivity constraint guarantees all network nodes are connected and thus all traffic demands can be accommodated. Note that the fiber tree length constraint is not considered due to short distances. The output of the heuristic approach consists of the $i$ ) established fiber trees and $i$ ) the possible routes each traffic demand can be assigned, and is passed as an input to an Integer Linear Programming (ILP)-based module (as shown in Figure 1).

Second step. An ILP model is used to solve the MRSA problem, which is formulated as follows: Given the set of fiber trees, the traffic demands, the possible route assignment per traffic demand and the set of modulation formats, we assign a modulation format and a route for each traffic demand and allocate the required frequency slot units with the objective of minimizing the maximum frequency slot unit (FSU) utilized among all links and/or the overall network spectrum utilization. The MRSA in filterless networks is subject to the following constraints:

- $\quad$ Each traffic demand is assigned exactly one route and one modulation format.

- Each traffic demand is assigned a sufficient number of FSUs, with a starting FSU and an ending FSU, guaranteeing FSU's contiguity constraint (consecutiveness of FSUs) and continuity constraint (an FSU on a link is assigned to only one demand). Note that the number of FSUs per demand is a variable in the problem as it depends on demand's bandwidth requested and the modulation format assigned and thus

- If an FSU is occupied on a link of a given fiber tree to accommodate a demand, the FSU is considered reserved on all links of the fiber tree, due to the broadcast nature of filterless networks, and thus cannot be assigned to any other demand passing through the links of the fiber tree.

Table 1 Characteristics of Transponders [5]

We consider two coherent transponders with Dual-Polarization (DP) that operate at line rates of 100 and 400 Gbps. Their characteristics are described in Table 1 . We assume a spectral granularity of $12.5 \mathrm{GHz}$ FSU, and consider 200 FSUs (i.e., a total of $2.5 \mathrm{THz}$ ) are available on each fiber, also including 1 FSU guard band between the optical channels.

\section{NETWORK AND TRAFFIC MODELS}

In this study we consider a metro topology similar to the one in Figure $\mathbf{2}$ as recommended in the context of Metro-HAUL project [10], consisting of 4 categories of nodes: the Access-Metro Edge Nodes (AMENs), the Metro-Core Edge Nodes (MCENs), the Metro Nodes (MN), and the Access Nodes (ANs). The AN is where traffic of fixed and mobile end-users is aggregated. The AMEN represents a central office where the access head-ends and the metro network interfaces are located. We assume the AMEN supports multiple access technologies and aggregates traffic from ANs. The MN represents a purely transport metro node. The MCEN is where the metro head-ends and the core network interfaces are located.
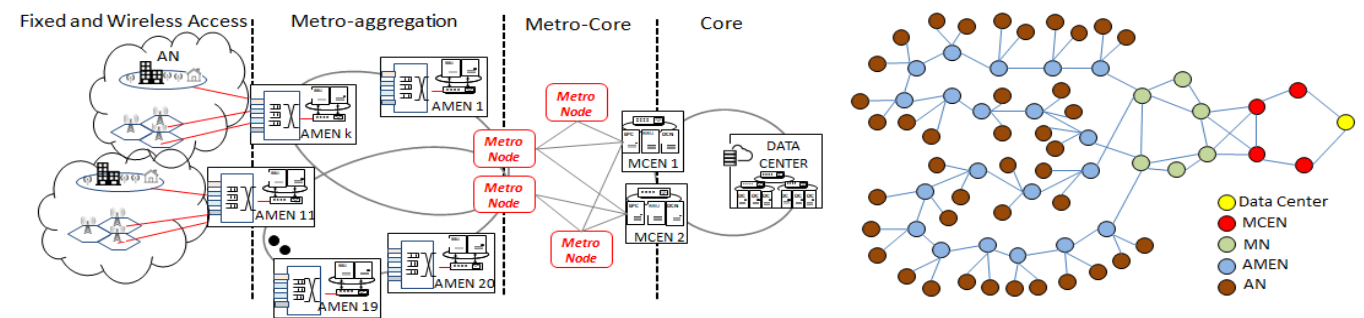

Figure 2 A schematic representation of the network topology showing the 4 network levels (left) and the network topology considered in our study (right). 
The AMENs and MCENs serve as mini and regional data centers, respectively, i.e., they are equipped with computing and storage capabilities and provide latency-stringent and/or bandwidth-hungry services (e.g., 4K Video on Demand delivery, Virtual Reality) requested by end-users. We consider 40 ANs, 20 AMENs, 6 MNs and 2 MCENs. The ANs are interconnected to AMENs in tree-like topologies. The AMENs are interconnected in a ring topology to MNs, which are interconnected in a meshed topology to MCENs. We consider a one bidirectional fiber where each fiber direction is used either for upstream or downstream traffic. Moreover, we consider 4 different network deployments, namely;

1) Active-Photonic, where all nodes are equipped with WSS-based ROADMs.

2) Filterless, a fully-filterless solution where both AMENs and MNs are assumed to be filterless.

3) Filterless MNs, a semi-filterless where only the MNs are considered to be filterless nodes whereas other network nodes are active.

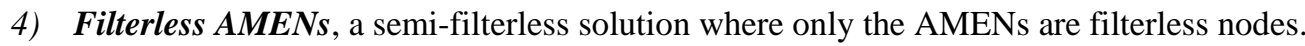

We compare the proposed network deployments in a context of a Video-on-Demand service, being one of the most bandwidth-demanding services. We assume AMENs and MCENs are equipped with caches storing video contents, where first-tier popular contents are stored at AMENs and second-tier popular contents are stored at MCENs, and then generate traffic accordingly. We assume each AN to request $150 \mathrm{Gbps}$ of traffic on average (equivalent to 10,000 video content requests with an average bit-rate of $15 \mathrm{Mbps}$ per request), summing up to a total of $6 \mathrm{Tbps}$ of overall network traffic. We also assume contents are delivered to ANs from the nearest location they are cached, i.e., either from the nearest AMEN or the nearest MCEN.

\section{ILLUSTRATIVE AND NUMERICAL RESULTS}

In this section we present illustrative numerical results, showing the formation of the fiber-trees in the proposed network solutions (i.e., the output of the heuristic approach for filterless network design) and comparing the four proposed network solutions in terms of spectrum utilization and equipment cost.

Fiber Trees Establishment: Figure 3 illustrates the network topology for the Active Photonic and the filterless solutions. For each of the Filterless, Filterless MNs and Filterless AMENs network deployments we obtained 2 fiber trees. The 2 fiber trees (T1 and T2) in the Filterless deployment span all network levels and connect the AMENs to the MCENs. In the Filterless MNs deployment, the 2 fiber trees guarantee network connectivity between MNs and MCENs, while in the Filterless AMENs deployment the 2 formed fiber trees create a 'horseshoe' topology (to avoid laser-loops), connecting AMENs of the same ring to two different MNs.

Now we compare the spectrum usage of the 4 network deployments in terms of cost, the maximum allocated spectrum slot number ( $\max F S U$ ), and the overall network spectrum utilization (total spec.), i.e., the total number of FSUs utilized.

Cost Comparison: Figure 4(a) shows the cost comparison of the considered network deployments, where arbitrary cost units (a.u.) obtained from Ref. [3] corresponding to the cost of nodes (WSSs and couplers) are assumed. Results show that filterless and semi-filterless solutions have significantly lower cost as active devices are replaced with passive components, however, for the filterless MNs network deployment, the cost is relatively high with respect to the other filterless network deployments due to the little penetration of passive components (only at MNs) and to the utilization of WSSs at all AMENs.

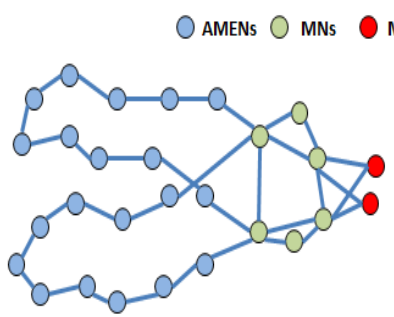

Active Photonic

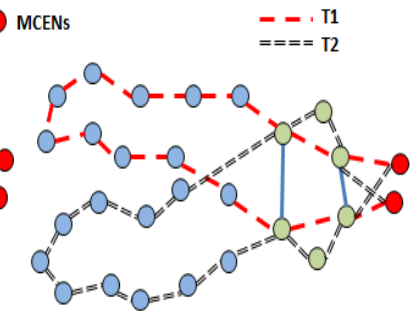

Filterless

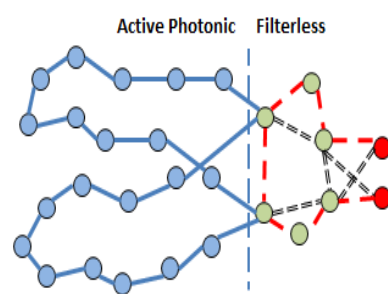

Filterless MNs

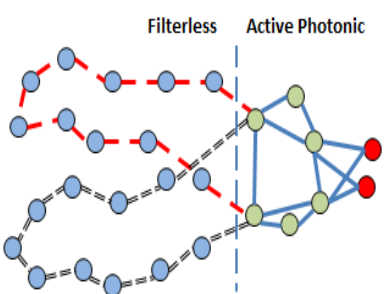

Filterless AMENs

Figure 3 (From left to right) The network topology for the Active Photonic and the fiber tree formation for the Filterless, the Filterless MN and the Filterless AMENs. Note that the ANs are not sketched as they act as leaves and belong to the same fiber.

Spectrum Utilization Comparison: Figure 4(b) and 4(c) show max FSU and total spec. of the four network deployments. On one hand, we see that the max. FSU values of the filterless solutions are comparable to that of the active photonic (only around 15\% difference), while on the other hand, the total spec. values of the filterless and semi-filterless solutions are significantly higher (around 2 to 3 folds) than that of the active photonic solution. This reveals that the comparable max FSU values are a result of congestion of the links which are forced to carry most of the traffic from the MCEN to the nearest AMEN of the AMENs ring. In other words, the high max FSU values are not due to widespread congestion among all network links, but only because few links suffer of high utilization. This also shows the max FSU cannot be considered a reliable metric to measure the overall spectrum utilization in hierarchical ring-based metro networks, where specific links are forced to carry most of the traffic. As for the total 

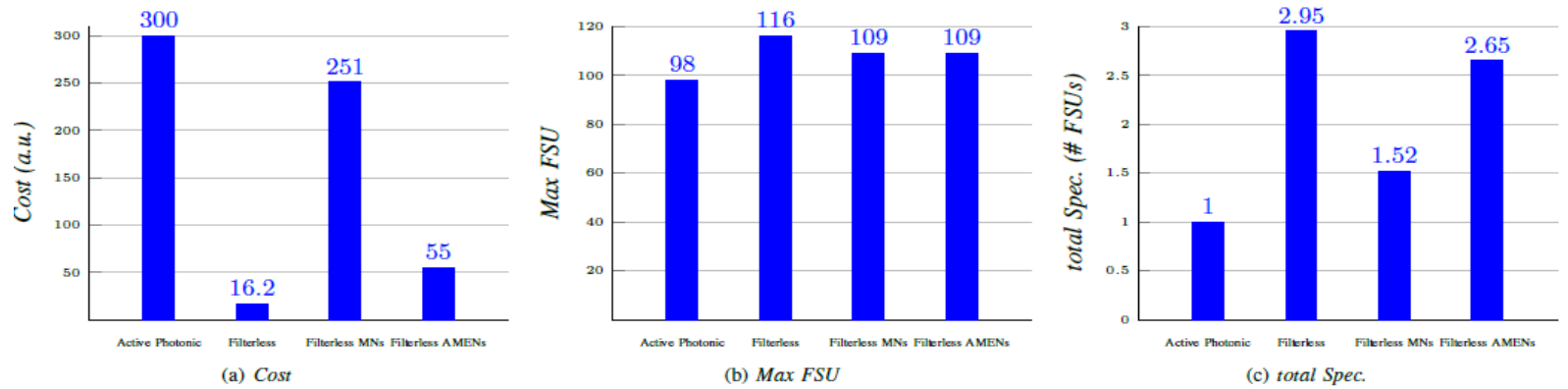

Figure 4 Comparison of (a) cost, (b) Max FSU and (c) total Spec. (normalized to that of Active Photonic).

spec., we see that the fully-filterless solution has the worst performance (highest amount of required FSUs). This is due to the length of the fiber trees which span all the hierarchical levels of the network causing excessive signal broadcast. Similarly, filterless AMENs solution shows a high total spec. with only a slight improvement with respect to the fully-filterless in spite the MNs being equipped with filters. This is because the presence of filters at the MNs network level helps reducing the spectrum utilization by preventing signal broadcast among the MNs, but then high spectrum is utilized once the signal propagates into the AMEN rings due to the filterless fiber tree connecting the AMENs. As for the Filterless MNs solution, it shows the best performance in terms of spectrum utilization (lowest total spec.) as the AMENs are equipped with filters, and thus no signal propagation of a request occurs beyond the AMEN which to which the destination-AN is connected. This also shows that the major part of the wasted spectrum is in the ring interconnecting the AMENs, and thus a strategic deployment of filters is motivated among AMENs more than among MNs.

In summary, results suggest that filterless AMENs, due to their location in the considered network topology, cause significant waste of spectrum. An alternative solution is to equip AMENs at strategic locations in the network topology with filters and wavelength blockers, such as the signal broadcast is narrowed. Moreover, due to the fact that the MNs are meshed-connected and that the traffic among them follows a specific path (e.g., incoming traffic from an MCEN is destined to nearest AMEN), spectrum waste reduction is possible among filterless MNs through the use of optical white box switches [6]. These aspects are currently under investigation to discover other possible advantages of filterless architectures in hierarchical metro networks.

\section{CONCLUSION}

In this paper we investigate the suitability of filterless optical network solutions in metropolitan area networks taking 'Metro-HAUL' metro network topology as a reference. We followed a two-steps approach for the design and resource allocation in filterless optical metro networks. We first developed a heuristic approach for the formation of fiber trees in the network and then solved the RSA problem via an ILP-based approach. We presented a comparative analysis between active photonic, filterless and semi-filterless network deployments in terms of spectrum utilization. On one hand, results show that due to the ring-based metro network topology the deployment of a fully-filterless solution yields the highest spectrum utilization ( 3 times that of an active photonic network) and thus is not suitable. On the other hand, the semi-filterless solutions show a promising performance as the presence of filters lessens the spectrum utilization (around 50\% more spectrum utilization).

\section{ACKNOWLEDGMENTS}

The work leading to these results has been supported by the European Community under grant agreement no. 761727 Metro-HAUL project funding.

\section{REFERENCES}

[1] C. Tremblay et al., "Filterless optical networks: a unique and novel passive WAN network solution." (2007)

[2] S. Khanmohamadi, et al. "Semi-filterless optical network: a cost-efficient passive wide area network solution with effective resource utilization." Communications and Photonics Conference and Exhibition, ACP. Asia. IEEE, 2011.

[3] C. Tremblay et al., "Passive filterless core networks based on advanced modulation and electrical compensation technologies," Telecommun. Syst., vol. 54, no. 2, July 2013.

[4] E. Archambault, et al. "Design and simulation of filterless optical networks: Problem definition and performance evaluation." Journal of Optical Communications and Networking 2.8 (2010).

[5] E. Archambault et al. "Routing and spectrum assignment in elastic filterless optical networks." IEEE/ACM Transactions on Networking 24.6 (2016).

[6] Z. Xu et al., "Flexible bandwidth allocation in filterless optical networks", IEEE Commun. Letters, vol. 19, no. 4, 2015.

[7] M. Furdek et al., "Programmable filterless network architecturebased on optical white boxes," in Optical Network Design and Modeling (ONDM), Cartagena, Spain, May 2016.

[8] L. Peterson, et al. "Central office re-architected as a data center." IEEE Communications Magazine 54.10 (2016).

[9] C. Tremblay, et al. "Agile Optical Networking: Beyond Filtered Solutions." Optical Fiber Communication Conference. Optical Society of America, 2018.

[10] https://metro-haul.eu/ 